\title{
Showing Fjortof's Theorem Does Not Apply for Defining Instability for Early Universe Thermodynamic Potentials. Asking If Nucleated Particles Result at/before Electro-Weak Era Due to Injection of Matter-Energy at the Big Bang?
}

\author{
Andrew Beckwith \\ Physics Department, College of Physics, Chongqing University, Huxi Campus, Chongqing, China \\ Email: abeckwith@uh.edu,rwill9955b@gmail.com
}

How to cite this paper: Beckwith, A. (2018) Showing Fjortofs Theorem Does Not Apply for Defining Instability for Early Universe Thermodynamic Potentials. Asking If Nucleated Particles Result at/before Electro-Weak Era Due to Injection of Matter-Energy at the Big Bang? Journal of High Energy Physics, Gravitation and Cosmolo$g y, 4,48-59$.

https://doi.org/10.4236/jhepgc.2018.41006

Received: September 11, 2017

Accepted: January 9, 2018

Published: January 12, 2018

Copyright $\odot 2018$ by author and Scientific Research Publishing Inc. This work is licensed under the Creative Commons Attribution International License (CC BY 4.0).

http://creativecommons.org/licenses/by/4.0/

\begin{abstract}
This paper uses the "Fjortoft theorem" for defining necessary conditions for instability. The point is that it does not apply in the vicinity of the big bang. We apply this theorem to what is called by T. Padmanabhan a thermodynamic potential which would become unstable if conditions for the applications of "Fjortoft's theorem" hold. In our case, there is no instability, so a different mechanism has to be appealed to. In the case of vacuum nucleation, we argue that conditions exist for the nucleation of particles as of the electroweak regime, due to injecting material from a node point, in spacetime. This regime of early universe creation coexists with the failure of applications of "Fjortoft" theorem in such a way as to give necessary and sufficient conditions for matter creation, in a way similar to the Higgs Boson.
\end{abstract}

\section{Keywords}

Fjortoft Theorem, Thermodynamic Potential, Matter Creation, Higgs Boson

\section{Introduction}

We first start off with a review of the classical Fjortoft theorem [1] and from there apply it to an early universe thermodynamic potential described by T. Padamanabhan [2] in Dice 2010. The objective will be to show that one can come 
up with a first principle creation of nucleated "particles", likely from a semi classical stand point which can be introduced by the creation of mass without appealing directly to the Higgs Boson in the first place. That is due to the fact that the Fjortoft theorem does not apply. There is an inflection point for the speed up of acceleration of the universe which exists one billion years ago for reasons which we will introduce in this manuscript. But no such inflection point exists at the origin of the big bang itself, or at the beginning of the electroweak era.

\section{Describing the Fjortoft Theorem}

From [1] we have that the theorem to be considered should be written up as follows, namely, look at

Fjortoft theorem:

A necessary condition for instability is that if $Z_{*}$ is a point in spacetime for which $\frac{\mathrm{d}^{2} U}{\mathrm{~d} z^{2}}=0$ for any given potential $U$, then there must be some value $z_{0}$ in the range $z_{1}<z_{0}<z_{2}$ such that

$$
\left.\frac{\mathrm{d}^{2} U}{\mathrm{~d} z^{2}}\right|_{z_{0}} \cdot\left[U\left(z_{0}\right)-U\left(z_{*}\right)\right]<0
$$

For the proof, see [1] and also consider that the main discussion is to find instability in a physical system which will be described by a given potential $U$. Next, we will construct in the boundary of the EW era, a way to come up with an optimal description for $U$

\section{Constructing an Appropriate Potential for Using Fjortoft Theorem in Cosmology for the Early Universe Cannot Be Done. We Show Why}

To do this, we will look at Padamanabhan [2] and his construction of (in Dice 2010) of thermodynamic potentials he used to have another construction of the Einstein GR equations. To start, Padamanabhan [2] wrote:

If $P_{c d}^{a b}$ is a so called Lovelock entropy tensor, and $T_{a b}$ a stress energy tensor

$$
\begin{aligned}
& \begin{aligned}
U\left(\eta^{a}\right) & =-4 \cdot P_{a b}^{c d} \nabla_{c} \eta^{a} \nabla_{d} \eta^{b}+T_{a b} \eta^{a} \eta^{b}+\lambda(x) g_{a b} \eta^{a} \eta^{b} \\
& =U_{\text {gravity }}\left(\eta^{a}\right)+U_{\text {matter }}\left(\eta^{a}\right)+\lambda(x) g_{a b} \eta^{a} \eta^{b}
\end{aligned} \\
& \Leftrightarrow U_{\text {matter }}\left(\eta^{a}\right)=T_{a b} \eta^{a} \eta^{b} ; U_{\text {gravity }}\left(\eta^{a}\right)=-4 \cdot P_{a b}^{c d} \nabla_{c} \eta^{a} \nabla_{d} \eta^{b}
\end{aligned}
$$

We now will look at

$$
\begin{aligned}
& U_{\text {matter }}\left(\eta^{a}\right)=T_{a b} \eta^{a} \eta^{b} ; \\
& U_{\text {gravity }}\left(\eta^{a}\right)=-4 \cdot P_{a b}^{c d} \nabla_{c} \eta^{a} \nabla_{d} \eta^{b}
\end{aligned}
$$

So happens that in terms of looking at the partial derivative of the top (2) Equation, we are looking at

$$
\frac{\partial^{2} U}{\partial\left(\eta^{a}\right)^{2}}=T_{a a}+\lambda(x) g_{a a}
$$


Thus, we then will be looking at if there is a specified $\eta_{*}^{a}$ for which the following holds.

$$
\begin{aligned}
& {\left[\frac{\partial^{2} U}{\partial\left(\eta^{a}\right)^{2}}=T_{a a}+\lambda(x) g_{a a}\right]_{\eta_{0}^{a}} *\left[-4 \cdot P_{a b}^{c d}\left(\nabla_{c} \eta_{0}^{a} \nabla_{d} \eta_{0}^{b}-\nabla_{c} \eta_{*}^{a} \nabla_{d} \eta_{*}^{b}\right)\right.} \\
& \left.+T_{a b} \cdot\left[\eta_{0}^{a} \eta_{0}^{b}-\eta_{*}^{a} \eta_{*}^{b}\right]+\lambda(x) g_{a b} \cdot\left[\eta_{0}^{a} \eta_{0}^{b}-\eta_{*}^{a} \eta_{*}^{b}\right]\right]<0
\end{aligned}
$$

What this is saying is that there is no unique point, using this $\eta_{*}^{a}$ for which (5) holds. Therefore, we say there is no official point of instability of $\eta_{*}^{a}$ due to (4). The Lagrangian structure of what can be built up by the potentials given in (4) with respect to $\eta_{*}^{a}$ mean that we cannot expect an inflection point with respect to a $2^{\text {nd }}$ derivative of a potential system. Such an inflection point designating a speed up of acceleration due to DE exists a billion years ago [3]. Also note that the reason for the failure for (5) to be congruent to (1) is due to

$$
\left[\frac{\partial^{2} U}{\partial\left(\eta^{a}\right)^{2}}=T_{a a}+\lambda(x) g_{a a}\right] \neq 0 \text {, for } \forall \eta_{*}^{a} \text { choices }
$$

To use Equation (6) properly, we use the material, in our reasoning from [4] [5] [6] [7] [8]. How and why can we do this?

We state that (6) tells us is that there is an embedding structure for early universe geometry, some of which may take the form of the following diagram.

\section{Working with a Way to Achieve Energy Injection into the Universe, without Appealing to Fjortoft Theorem for Alleged Instabilities Starting from Padmanabhan Thermodynamic Potential Terms}

Padmanabhan [2] introduced the following discussion as to entropy, namely starting with energy, we have

$$
E=\frac{1}{2} k_{B} \int \mathrm{d} n T_{l o c}
$$

And the $n$ value as in (7) is given by

$$
\mathrm{d} n=32 \pi \cdot P_{c d}^{a b} \cdot \varepsilon_{a b} \cdot \varepsilon^{c d} \cdot \mathrm{d} A
$$

where $P_{c d}^{a b}$ is a so called Lovelock entropy tensor, and $\varepsilon_{a b}$ a bi normal on the codimension -2 cross section, and then entropy is stated to be

$$
S \propto \int_{\partial V} \mathrm{~d} n \propto \int_{\partial V} 32 \pi \cdot P_{c d}^{a b} \cdot \varepsilon_{a b} \cdot \varepsilon^{c d} \sqrt{\sigma} \mathrm{d}^{D-2} x
$$

The end result, is that energy is induced via the temperature $T_{l o c}$, while [2]

$$
T_{l o c}=N \frac{a^{\mu} n_{\mu}}{2 \pi}=\text { local acceleration temperature }
$$

Also, the change in $\mathrm{n}$ can be given by, if $l_{P}$ is the Planck's length value [2]

$$
\Delta n=\sqrt{\sigma} \mathrm{d}^{2} x / l_{P}
$$


Looking at (9) and (11) we state that the change in number count given in (11) is really a holographic surface phenomena, with $\mathrm{N}$ defined [2]

$$
N=E /\left[(1 / 2) k_{B} T\right]
$$

The upshot is that we can, as implied by $\mathrm{Ng}$ [4] easily reference a change in entropy via [4] [5] [6]

$$
S \sim n
$$

While having a change in $\mathrm{n}$ as due to a change in the spatial surface of spacetime as given in (11), we have to realistically infer that the local acceleration temperature (10) is from another pre universe construction and that local instability is ruled out by (5) and (6). This leads us to ask as to what would be an acceptable way to form the formation of mass, i.e. say the mass of a graviton, via external factors introduced into our universe prior to the Electroweak era, in cosmology. To do that, look at if there are two branes on the $A d S_{5}$ space-time so that with one moving and one stationary, we can look at Figure 1 as background as to introduce such external factors in our present space-time universe during its initial expansion phase.

\section{Fall out from Adopting Figure 1 and That Due to No Instability in the Padamanabhan Supplied Potentials. i.e. a Way to Obtain Graviton Mass via a Root Finding Method}

Using [7], what we find is that there are two branes on the $A d S_{5}$ space-time so that with one moving and one stationary, we can look at Figure 1 which is part of the geometry used in the spatial decomposition of the differential operator

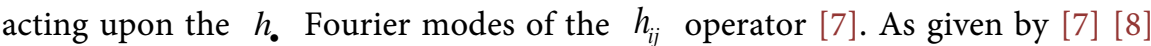
we have that

$$
\left[\partial_{t}^{2}+k^{2}-\partial_{y}^{2}+\frac{3}{y} \cdot \partial_{y}\right] h_{\bullet}=0
$$

Using [8] (and also [7]) the solution to (14) above takes the form of having

$$
h_{\bullet}=H_{i j}=e_{i j} \cdot \exp [i \cdot \omega \cdot t] \cdot(m \cdot y)^{2} \cdot A \cdot J_{2}(m \cdot y)
$$

$e_{i j}$ is a polarization tensor, and the function $J_{2}(m y)$ is a $2^{\text {nd }}$ order Bessel function [9]. A generalization offered by Durrer et al. [7] [8] leads to

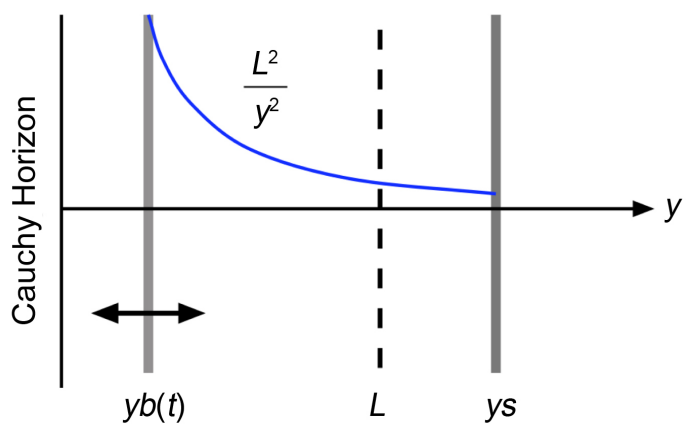

Figure 1. Brane world construction, as seen from [7] [8]. 


$$
h=\left\{\exp [i \cdot \omega \cdot t] \cdot(m \cdot y)^{2} \cdot A \cdot J_{2}(m \cdot y)\right\} \cdot\left(1+\frac{\pi}{4} \cdot(m \cdot \ell)^{2}\right)
$$

With the factor of $\left(1+\frac{\pi}{4} \cdot(m \cdot \ell)^{2}\right)$ coming in due to a boundary condition upon the wall of a brane put in, i.e. looking at [7], with the right hand side of (16) due to a domain wall tension of a brane.

$$
-2 \cdot \partial_{y} H_{i j}=\kappa_{5} \cdot \pi_{i j}^{(T)} \rightarrow 0
$$

This will be in our example set as not equal to zero, in the right hand side, but equal to an extremely small parameter, namely

$$
\left.\partial_{y} H_{i j}\right|_{y=y b}=\kappa_{5} \cdot \pi_{i j}^{(T)} \sim \xi^{+}
$$

With this turned into

$$
\left.\partial_{y} h\right|_{y=y b} \sim \delta^{+}
$$

The right hand side of (19) represents very small brane tension, which is understandable. Then using [7] [8] [10], i.e.

$$
\left.\partial_{y} h\right|_{y=y b}=\left.\partial_{y}\left\{\exp [i \omega t] \cdot(m y)^{2} \cdot A \cdot J_{2}(m y)\right\} \cdot\left(1+\frac{\pi}{4} \cdot(m \cdot \ell)^{2}\right)\right|_{y=y b} \sim \delta^{+}
$$

and

$$
J_{2}(m y)=\frac{(m y)^{2}}{2^{2} \times 2 !} \cdot\left(1-\frac{(m y)^{2}}{2^{2} \times 3}+\frac{(m y)^{4}}{2^{4} \times 2 ! \times 3 \times 4}-\frac{(m y)^{6}}{2^{6} \times 4 ! \times 3 \times 4 \times 5}+\cdots\right)
$$

The upshot is, that afterwards,

$$
\begin{aligned}
& \frac{(m y)^{4}}{2^{2} \times 2 !} \cdot \frac{1}{y}\left[\left(1-\frac{(m y)^{2}}{2^{2} \times 3}+\frac{(m y)^{4}}{2^{4} \times 2 ! \times 3 \times 4}-\frac{(m y)^{6}}{2^{6} \times 4 ! \times 3 \times 4 \times 5}+\cdots\right)\right. \\
& \left.-\left(\frac{2 \cdot(m y)^{2}}{2^{2} \times 3}+\frac{4 \cdot(m y)^{4}}{2^{4} \times 2 ! \times 3 \times 4}-\frac{6 \cdot(m y)^{6}}{2^{6} \times 4 ! \times 3 \times 4 \times 5}+\cdots\right)\right] \\
& =\frac{\delta^{+} \cdot \exp [\mp i \omega t]}{A} \cdot\left[1-\frac{\pi}{4} \cdot(m \cdot \ell)^{2}\right]
\end{aligned}
$$

Should the term

$$
\frac{\delta^{+} \cdot \exp [\mp i \omega t]}{A} \cdot\left[1-\frac{\pi}{4} \cdot(m \cdot \ell)^{2}\right] \underset{\delta^{+} \rightarrow 0}{\longrightarrow} 0
$$

Then, (22) is acting much as in [7] and [8], whereas, one is recovering a simple numerical exercise as to obtain a suitable solution as given by (18), and (19) due to [7] [8] where the domain tension of the brane vanishes. The novelty as to this approach given in (22) is to obtain a time dependent behavior of the mass of the graviton,

$$
(m y)=f(t) \Leftrightarrow m \equiv \frac{f(t)}{y}
$$


In doing all of this, keep in mind the mathematical information given in [10] which is repeatedly used in [7] and [8].

Needless to say, (22) can only be solved for, numerically, i.e. fourth order polynomial solutions for quartic equations still give over simplified dynamics, especially if (24) holds, and makes things more complicated. This is all being done to keep fidelity with respect to [11] and [12] as a possible feature of brane world dynamics as reflected in [11] [12], as well as certain issues brought up in [13], as to what is a semi classical argument can obtain a usually quantum result. It also would allow for eventually understanding if entropy can also be stated in terms of gravitons alone in early universe models as was proposed by Kiefer \& Starobinsky, et al. [14]. Finally, it would address if QM is embedded in a larger deterministic theory as advocated by 't Hooft [9], as well as degrees of freedom in early universe cosmology as brought up by Beckwith in Dice 2010 [15]. We argue that making this step is consistent with keeping the value of Planck's constant uniform in spite of Avessans theories suggesting it vary in time [16]. To do this, we make extensive use of [17] and [18].

It is now then time to do a recap and to organize how such speculation can be vetted using experimental procedures. To do this were cap what can be said about traces massive gravitons can be detected, prior to our conclusion which may lead to an explanation of the following entropy formula [19]

$$
S_{g w}=V \cdot \int_{v 0}^{v 1} r(v) \cdot v^{2} \mathrm{~d} v \cong\left(10^{29}\right)^{3} \cdot\left(H_{1} / M_{P}\right)^{3 / 2} \approx 10^{87}-10^{88}
$$

This is a bridge to future projects which should be kept in mind, i.e., could our formulation of graviton physics lead to identification of gravitons, in the early universe as the main driver of graviton physics being the primary entropy generator as suggested by [19]?

\section{Conclusions: Semiclassical Method of Obtaining Graviton Mass Procedure Cannot Be Ruled out, and It Impacts Relic GW Searches}

First of all, we review the details of a massive graviton imprint upon $h_{i j}$, and then we will review the linkage between that and certain limits upon $h_{\bullet}$ as read from Hinterbichler [20], if $r=\sqrt{x_{i} x_{i}}$, and we look at a mass induced $h_{i j}$ suppression factor put in of $\exp (-m \cdot r)$, then if

$$
\begin{aligned}
h_{00}(x)=\frac{2 M}{3 M_{\text {Planck }}} \cdot \frac{\exp (-m \cdot r)}{4 \pi \cdot r} & h_{0 i}(x)=0 \\
h_{i j}(x)= & {\left[\frac{M}{3 M_{\text {Planck }}} \cdot \frac{\exp (-m \cdot r)}{4 \pi \cdot r}\right] } \\
& \cdot\left(\frac{1+m \cdot r+m^{2} \cdot r^{2}}{m^{2} \cdot r^{2}} \cdot \delta_{i j}-\left[\frac{3+3 m \cdot r+m^{2} \cdot r^{2}}{m^{2} \cdot r^{4}}\right] \cdot x_{i} \cdot x_{j}\right)
\end{aligned}
$$


Here, we have that these $h_{i j}$ values are solutions to the following equation, as given by [21], with $\mathrm{D}$ a dimensions value put in.

$$
\left(\partial^{2}-m^{2}\right) h_{\mu v}=-\kappa \cdot\left[T_{u v}-\frac{1}{D-1} \cdot\left(\eta_{u v}-\frac{\partial_{\mu} \partial_{v}}{m^{2}}\right) \cdot T\right]
$$

To understand the import of the above equations, set

$$
\begin{aligned}
& M=10^{50} \times 10^{-27} \mathrm{~g} \equiv 10^{23} \mathrm{~g} \propto 10^{61}-10^{62} \mathrm{eV} \\
& M_{\text {Plank }}=1.22 \times 10^{28} \mathrm{eV}
\end{aligned}
$$

We should use the $m_{\text {massive-graviton }} \sim 10^{-26} \mathrm{eV}$ value in (29) above.

In reviewing what was said about (27), (28) we should keep in mind the overall Fourier decomposition linkage between $h_{\bullet}, h_{i j}$ which is written up as

$$
h_{i j}(t, x ; k)=\frac{1}{(2 \pi)^{3 / 2}} \int \mathrm{d}^{3} k \sum_{\bullet++, \otimes} \mathrm{e}^{i k \cdot x} e_{i j}^{\bullet} h_{\bullet}(t, y ; k)
$$

The bottom line is that a simple decomposition involving a basis in two polarization states, of,$+ \otimes$ will have to be amended and adjusted, if one is looking at massive graviton states.

In addition further developments as to (31) could influence giving a semi classical interpretation as to entropic origins of gravity, along the lines brought up by both 't Hooft, indirectly [9], and Lee [22] directly.

The experimental gravity considerations are covered in [23] [24] [25] [26], and the idea should be especially in our work to examine if [23] and [25] in terms of gravity are adhered to. As these are LIGO projects, we should be looking to see if what we are doing contravenes or backs the post Newtonian approximations of physics, so brought up.

Reference [25] is a must to review. In it, Corda reviews GR tests and our document must not contravene these basics. Can we obtain through our representation of gravitons, confirmation, or refutation of if the data sets are in adherence, or partially refute General Relativity.

As far as [26], in terms of quantum cosmology, it is another similar parallel development to the ideas raised here. I urge readers to investigate it.

Finally, in lieu of [27] the author urges readers to look at Appendix A, which is a summary of what the author views as to what would be foundational investigation of gravity, and to see if it can be made in adherence to GR, and possibly quantized.

The author urges readers to look at [27], as well as [28] by Kieffer, and that when seeing Appendix A, that this is a schematic the author believes would be appropriate for an investigation to confirm if Gravity could be derived as having quantum roots, which in turn would affect the viability of presumed quantum gravity. As well as issues brought up in what is called later on the cited references [29] [30] [31] [32] [33] in the Appendix A.

\section{Acknowledgements}

This work is supported in part by National Nature Science Foundation of China 
grant No110752.

\section{References}

[1] Pringle, J. and King, A. (2007) Astrophysical Flows. Cambridge University Press, New York.

[2] Padmanabhan, T. (2011) Lessons from Classical Gravity about the Quantum Structure of Space-Time. Journal of Physics: Conference Series, 306, Article ID: 01200. http://iopscience.iop.org/1742-6596/306/1/012001

[3] Beckwith, A. (2011) Identifying a Kaluza Klein Treatment of a Graviton Permitting a Deceleration Parameter Q $(Z)$ as an Alternative to Standard DE. Journal of Cosmology, 13, 1-15. http://journalofcosmology.com/BeckwithGraviton.pdf

[4] Ng, Y.J. (2008) Holographic Foam, Dark Energy and Infinite Statistics. Physics Letters, Section B: Nuclear, Elementary Particle and High-Energy Physics, 657, 10-14.

[5] Ng, Y.J. and van Dam, H. (2000) On Wigner's Clock and the Detectability of Spacetime Foam with Gravitational-Wave Interferometers. Physics Letters B, 477, 429-435.

[6] Ng, Y.J. (2012) https://cgc.physics.miami.edu/Miami2012/Ng.pdf

[7] Durrer, R., et al. (2009) Dynamical Casmir Effect for Gravitons in Bouncing Brane Worlds. Physical Review D, 76, Article ID: 104014.

http://www.scribd.com/doc/79001898/Ruth-Durrer-and-Marcus-Ruser-Thedynami cal-Casimir-effect-in-braneworlds

[8] Durrer, R. (2017) Branewords. http://arxiv.org/pdf/hep-th/0507006v1.pdf

[9] 't Hooft, G. (1999) Quantum Mechanics as a Dissipative Deterministic System. http://arxiv.org/PS_cache/gr-qc/pdf/9903/9903084v3.pdf

[10] Bessel Functions and Hankel Functions: NIST. http://dlmf.nist.gov/10.2

[11] Steinhardt, P.J. and Turok, N. (2002) Cosmic Evolution in a Cyclic Universe. Physical Review D, 65, Article ID: 126003.

[12] Koury, J., Steinhardt, P.J. and Turkok, N. (2003) Inflation versus Cyclic Predictions for Spectral Tilt. Physical Review Letters, 91, Article ID: 161301.

[13] Cartier, C., Durrer, R. and Ruser, M. (2005) On Graviton Production in Braneworld Cosmology. Physical Review D, 72, Article ID: 104018.

[14] Kiefer, C., Polarski, D. and Starobinsky, A.A. (2000) Entropy of Gravitons Produced in the Early Universe. PRD, 62, 043518.

[15] Beckwith, A. (2011) How to Use the Cosmological Schwinger Principle for Energy Flux, Entropy, and "Atoms of Space-Time" to Create a Thermodynamic Space-Time and Multiverse. Journal of Physics. Conference Series, 306, Article ID: 012064. http://iopscience.iop.org/1742-6596/306/1;jsessionid=A05372A78C18D970BF35F40 A9A863B51.C

[16] Avessian, A.K. (2009) Planck's Constant Evolution as a Cosmological Evolution Test for the Early Universe. Gravitation and Cosmology, 15, 10-12. https://doi.org/10.1134/S0202289309010034

[17] Bruchholz, U. (2009) Derivation of Planck's Constant from Maxwell's Electrodynamics. Progress in Physics, 4, 67.

[18] Bruzchholz, U. (2009) Key Notes on a Geometric Theory of Fields. Progress in Physics, 2, 107-113.

[19] Giovannini, M. (2008) A Primer on the Physics of the Cosmic Microwave Background. World Press Scientific, Singapore. 
[20] Hinterbichler, K. (2012) Theoretical Aspects of Massive Gravity. http://arxiv.org/abs/1105.3735.pdf

[21] http://web.mit.edu/redingtn/www/netadv/Xgravitati.html

[22] Lee, J.-W. (2012) On the Origin of Entropic Gravity and Inertia. http://arxiv.org/abs/1003.4464

[23] Abbott, B.P., et al. (2016) Observation of Gravitational Waves from a Binary Black Hole Merger. Physical Review Letters, 116, Article ID: 061102. https://physics.aps.org/featured-article-pdf/10.1103/PhysRevLett.116.061102

[24] Corda, C. (2009) Interferometric Detection of Gravitational Waves: The Definitive Test for General Relativity. International Journal of Modern Physics D, 18, 2275-2282. https://doi.org/10.1142/S0218271809015904

[25] Abbot, B.P., et al. (2016) GW151226: Observation of Gravitational Waves from a 22-Solar-Mass Binary Black Hole Coalescence. Physical Review Letters, 116, Article ID: 241103. https://doi.org/10.1103/PhysRevLett.116.241103

[26] Halliwell, J. (1991) Quantum Cosmology. The ICTP Series in Theoretical Physics, Volume 7, Trieste, 18 June-28 July 1990, 515-599.

[27] Beckwith, A. (2017) History Lessons from the 5th Solvay Meeting, 1927. http://vixra.org/abs/1708.0399

[28] Kieffer, C. (2012) Quantum Gravity. 3rd Edition, Oxford Science Publications, Oxford University Press, Oxford.

[29] Dirac, P. (2001) Lectures on Quantum Mechanics. Dover Publications, Inc., New York.

[30] Ryden, B. (2017) Introduction to Cosmology. 2nd Edition, Cambridge University Press, Cambridge.

[31] Bacciagaluppi, G. and Valentini, A. (2009) Quantum Theory at the Crossroads: Reconsidering the 1927 Solvay Conference. Cambridge University Press, Cambridge.

[32] Omnes, R. (1999) Understanding Quantum Mechanics. Princeton University Press, Princeton.

[33] Omnes, R. (1994) The Interpretation of Quantum Mechanics. Princeton University Press, Princeton. 


\section{Appendix A}

From [27], a quote of would be first principles of how to quantize the Einstein field equations elucidated by first principles:

From [27] where we quote large parts of our text in [27].

XIX. A generalized problem to making quantization of the Einstein field equations elucidated by first principles. (This reflects our evaluation of [28]). i.e. the lectures on quantization of a classical Hamiltonian given by Dirac, in [29], pages 25-43 is ironically made more fraught by the requirement of extending the Hamiltonian i.e. if we have say $\phi_{a^{\prime}}$ as so called first class secondary constraints, page 25 of [29] we find that there is an inability to do the following, if we wish to transfer to quantum systems, we need to do the following, i.e. add to the initial classical Hamiltonian, $H_{T}$.

$$
\begin{aligned}
& H_{E}=H_{T}+v_{a^{\prime}} \cdot \phi_{a^{\prime}} \\
& \dot{g} \approx\left\{g, H_{E}\right\}
\end{aligned}
$$

Equation (A1), in a Poisson bracket formulation, was used by Dirac to transform to a set of quantization conditions, in pages 25 to 43 of. The problem is, that it is difficult to come up with constraint equations, as given in the top level of Equation (A1).

The following is easy to do, if you ignore constraints

$$
\begin{aligned}
& \frac{\mathrm{d}\langle P\rangle}{\mathrm{d} t}=-\frac{i}{\hbar}\langle\Psi|[P, H]| \Psi\rangle=-\frac{1}{i \hbar}\langle\Psi|[P, H]| \Psi\rangle \\
& \underset{\text { 3dim } \rightarrow 1 \operatorname{dim}}{\longrightarrow}-\int \Psi^{*} \cdot \frac{\mathrm{d} V(x)}{\mathrm{d} x} \cdot \Psi \mathrm{d} x \\
& \underset{\text { 3dim } \rightarrow \text { Any dim }}{\longrightarrow}\left\langle-\frac{\mathrm{d} V(x)}{\mathrm{d} x}\right\rangle \sim\langle-\vec{\nabla} V\rangle \equiv F \text { (force) }
\end{aligned}
$$

Try doing this, to have equivalence with Equation (A1) and match that with Equation (A2) the equations below given as (A3) to Equation (A4), i.e. what is so difficult is to put in a Hamiltonian system, for gravity, which is complicated. Worse than that, we do not have a quantum mechanical equivalent, and this due to the difficulties in terms of finding a quantum mechanical equivalent to the Poisson brackets $\left\{p_{N}, H\right.$ (Hamiltonian $\left.)\right\} \approx 0$ which is readily transferrable to the Friedman equation, i.e. so far a quantum bridge between quantized versions of Equation (A3) and Equation (A4) does not exist, right now. As we wrote in [27].

So as given in [27], as stated by Kieffer, [28] there is a relationship between a Hamiltonian form, $H$ (Hamiltonian), and a constraint equation, for momentum $p_{N}$, along the lines of

$$
p_{N} \&\left\{p_{N}, H(\text { Hamiltonian })\right\} \approx 0
$$

This is, according to Kieffer, the Poisson brackets, equivalent to the following.

What we are looking at is, if we set the Lapse function, $N$, as $=1$.

$$
\dot{a}^{2}=-1+a^{2} \cdot\left(\dot{\phi}^{2}+\frac{\Lambda}{3}+m^{2} \cdot \phi^{2}\right) \Leftrightarrow \ddot{\phi}^{2}+3 \frac{\dot{a}}{a} \cdot \dot{\phi}+m^{2} \phi=0
$$


Here, the $\phi$ is a scalar field (here, called a "homogeneous field"), $\mathrm{m}$ is a mass term, and $a$ the scale factor, and $\Lambda$ the cosmological constant. If $m$ is set equal to zero, this has a simple $m=0$ solution with

$$
\begin{aligned}
& p_{\phi}=a^{3} \cdot \dot{\phi}=\kappa=\text { const } \\
& \& \phi= \pm \frac{1}{2} \cdot \operatorname{arcosh} \frac{\kappa}{a^{2}}
\end{aligned}
$$

It cannot be solved analytically, if $m$ is not equal to zero. Now as to a general problem between the Solvay 1927 conference methods and the application to GR will be alluded to, next.

Dirac claims the bridge from Poisson brackets to the situation represented by Equation (A5) always involves a carefully set extended Hamiltonian situation, i.e. see his discussion in 33 to page 35 of [29]. The challenge would be to make those extensions somehow commensurate with Equation (A4) and Equation (A5).

Having said, this, we will next go to the problem of Quantum Geometrodynamics. Before going to it, a notice as to the problems of bridging to general relativity using conventional Quantum mechanics, will be raised as a bridge to the use of $H_{A D M} \Psi=0$ which makes a plausible bridge to the Fluid equation of general relativity, [30] but also a summary as to how and why the connection to the rest of general relativity is extremely difficult, i.e. the Friedman equation as seen in [30] has a classical analogue which cannot be linked to its general relativistic form, but the fluid equation of General relativity in [30] does have a Newtonian derivation yielding the exact same result in both Newtonian and GR physics. Hence, the quantum-classical bridge as exemplified by Equation (A5) works for the fluid equation, but would not work for the GR Friedman equation, since the Friedman equation classical would be the only bridge to the quantum result, using the Equation (A5) bridge. And of course, both the GR Friedman bridge plus the fluid cosmology bridge are both needed in the acceleration equation, i.e. from [30] the following cannot be linked to quantum mechanics, via Equation (A5), namely the acceleration equation of GR has

$$
\left(\frac{\ddot{a}}{a}\right)=-\frac{4 \pi G}{3 c^{2}} \cdot(\varepsilon+3 P)
$$

This requires two equations, namely,

$$
\begin{aligned}
& \left(\frac{\ddot{a}}{a}\right)=-\frac{4 \pi G}{3 c^{2}} \cdot(\varepsilon+3 P) \\
& \text { due to } \dot{\varepsilon}+3\left(\frac{\dot{a}}{a}\right) \cdot(\varepsilon+3 P)=0 \\
& \text { and } \dot{a}^{2}=\frac{8 \pi G}{3 c^{2}} \cdot \varepsilon a^{2}-\frac{\kappa c^{2}}{R_{0}^{2}} \text { (GR-Friedman) }
\end{aligned}
$$

as opposed to, if $U=$ const.

$$
\dot{a}^{2}=\frac{8 \pi G}{3 c^{2}} \cdot \rho a^{2}+\frac{2 U}{r_{s}^{2}}(\text { Newtonian-Friedman })
$$


The derivation of the acceleration equation for GR, using the two equations cited is in [30], page 60 .

In addition we will derive the Fluid equation also used, which is the same form used in Equation (A5) making a linkage to relativity and quantum mechanics, possible, if one uses the following steps, as given on page 59 of [30], i.e. If exists a commoving radius $r_{S}$.

We then will get a clean derivation of the so called fluid equation, used in Cosmology. This fluid equation, which has the same form used in both GR and Newtonian physics may be in principle linkable to the quantization program outlined in Equation (A5). So with that, we go to the interactions given in Equation (A8) below.

$$
\begin{aligned}
& V(t)=\text { Volume }(\text { universe })=\frac{4 \pi r_{s}^{3} a^{3}}{3} \\
& \& \dot{V}=V \cdot\left(\frac{3 \dot{a}}{a}\right) \\
& E=V(t) \cdot \varepsilon(t) \\
& \dot{E}=V \cdot\left(\dot{\varepsilon}+\frac{3 \dot{a}}{a} \varepsilon\right) \\
& \quad \& \text { First law thermo(universe) } \\
& \dot{E}+P \dot{V}=0 \\
& \Rightarrow V \cdot\left(\dot{\varepsilon}+\frac{3 \dot{a}}{a} \varepsilon+\frac{\dot{a}}{a} P\right)=0 \\
& \Rightarrow \dot{\varepsilon}+\frac{3 \dot{a}}{a} \varepsilon+\frac{\dot{a}}{a} P=0
\end{aligned}
$$

The GR and classical physics forms of the fluid equation, so derived, in Equation (A8) and the results at the bottom of Equation (A7) would allow us to make connection, with a lot of work to the sort of reasoning used in Equation (A5) above, but due to the difference in the Friedman equation, in classical and GR form, as noted in Equation (A6), it would be using the Solvay methods, extremely difficult to make connection between an acceleration equation, using scale factors, as given in Equation (A6) and Equation (A7) with the Equations (A5) (58) connection between classical and quantum mechanics with respect to an acceleration of the universe acceptable in both GR and quantum form.

We can state though that a bridge to the Fluid equation, as given in [27] Equation (A8) and Equation (A5) would at least in principle very doable. Having said that, let us now go to the ideas of Quantum Geometrodynamics, as far as their use and future prospects to the study of Solvay 1927 methods [31], and quantum gravity issues [28].

End of our quote from [27].

As a close to this, all this, in terms of quantum gravity should also keep in mind issues brought up in [31] and [32], in particular, quantum entanglement and how information is transferred in cosmology, i.e. the Geometrodynamics part of [28], as well as referencing issues brought up in [33]. 